\title{
Heartbeat: The gut microbiota and heart failure
}

\author{
Catherine M Otto, Kazem Rahimi
}

Patients with acute heart failure are at high risk of adverse clinical outcomes. Although elevated levels of trimethylamine N-oxide (TMAO), a gut-derived metabolite, have been shown to be independently associated with cardiovascular disease and poor outcomes in stable heart failure patients, their role in patients with acute heart failure is not well understood. In this issue of Heart, Suzuki and colleagues (see page 841 ) investigate this question further and show that the addition of TMAO to established multivariable risk models led to better stratification of acute heart failure patients' risk of in-hospital mortality (figure 1). However, the association was attenuated when renal function was added to the models.

In an accompanying editorial, Tang and Wilson (see page 813) provide an overview of the role of TMAO as a marker of risk in other clinical settings and comment that the complementary findings from this new analysis provide important insights as to how gut microbia may contribute to disease progression in HF. They speculate that TMAO may in fact have a causal role in HF deterioration. To this end, they conclude that "further studies are warranted to determine if circulating TMAO levels can serve as a guidance to tailor

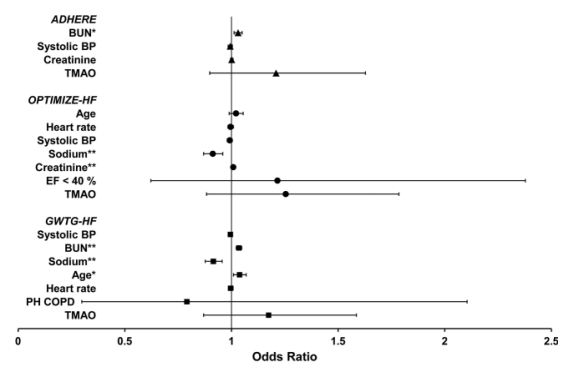

Figure 1 Forest plots to show binary logistic ORs (dots) and 95\% Cls (horizontal bars) for cardiac risk factors for multivariate models for the individual components of in-hospital mortality prediction algorithms of ADHERE (triangles), OPTIMIZE-HF (circles) and GWTG-HF (squares) registries with the addition of TMAO. BP, blood pressure; BUN, blood urea nitrogen; $\mathrm{COPD}$, chronic obstructive pulmonary disease; $\mathrm{EF}$, ejection fraction; $\mathrm{PH}$, past history; TMAO, trimethylamine $N$-oxide; ${ }^{*} \mathrm{p}<0.05$; ${ }^{* *} p<0.0005$.

Correspondence to Professor Catherine M Otto, Division of Cardiology, University of Washington, Seattle,WA 98195, USA; cmotto@u.washington.edu dietary modulation or therapeutic interdiction in either acute or chronic HF".

Multimodality imaging provides sophisticated measures of myocardial perfusion, sympathetic denervation and myocardial scarring, each of which has been proposed to predict the risk of ventricular arrhythmias in patients with ischemic heart disease. Rijnierse and colleagues (see page 832) performed a detailed study in 52 patients with left ventricular (LV) systolic dysfunction (ejection fraction 35\%) due to ischemic heart disease, with multimodality imaging at baseline followed by electrophysiological study (EPS) after placement of an implantable cardioverterdefibrillator (ICD) (figure 2). Hyperaemic myocardial blood flow (MBF) measured by positron emission tomography was the single best independent predictor or an inducible sustained ventricular arrhythmia on EPS. Interestingly, predictive value of hyperemic MBF was not improved by addition other risk markers including sympathetic denervation size, extent of myocardial scar, perfusion defect size, LV volumes or ejection fraction.

Commenting on this study in an editorial, Kaufmann and Stehli (see page 815) express surprise that data from the current study did not support the value of previously identified risk predictors, such as extent of scar tissue, and they suggest that further follow-up on this study cohort will be of interest because ventricular arrhythmias on EPS “may not be a perfect surrogate marker for sudden cardiac death and ICD discharges". In addition, they caution that "so far there is no solid evidence from prospective randomised
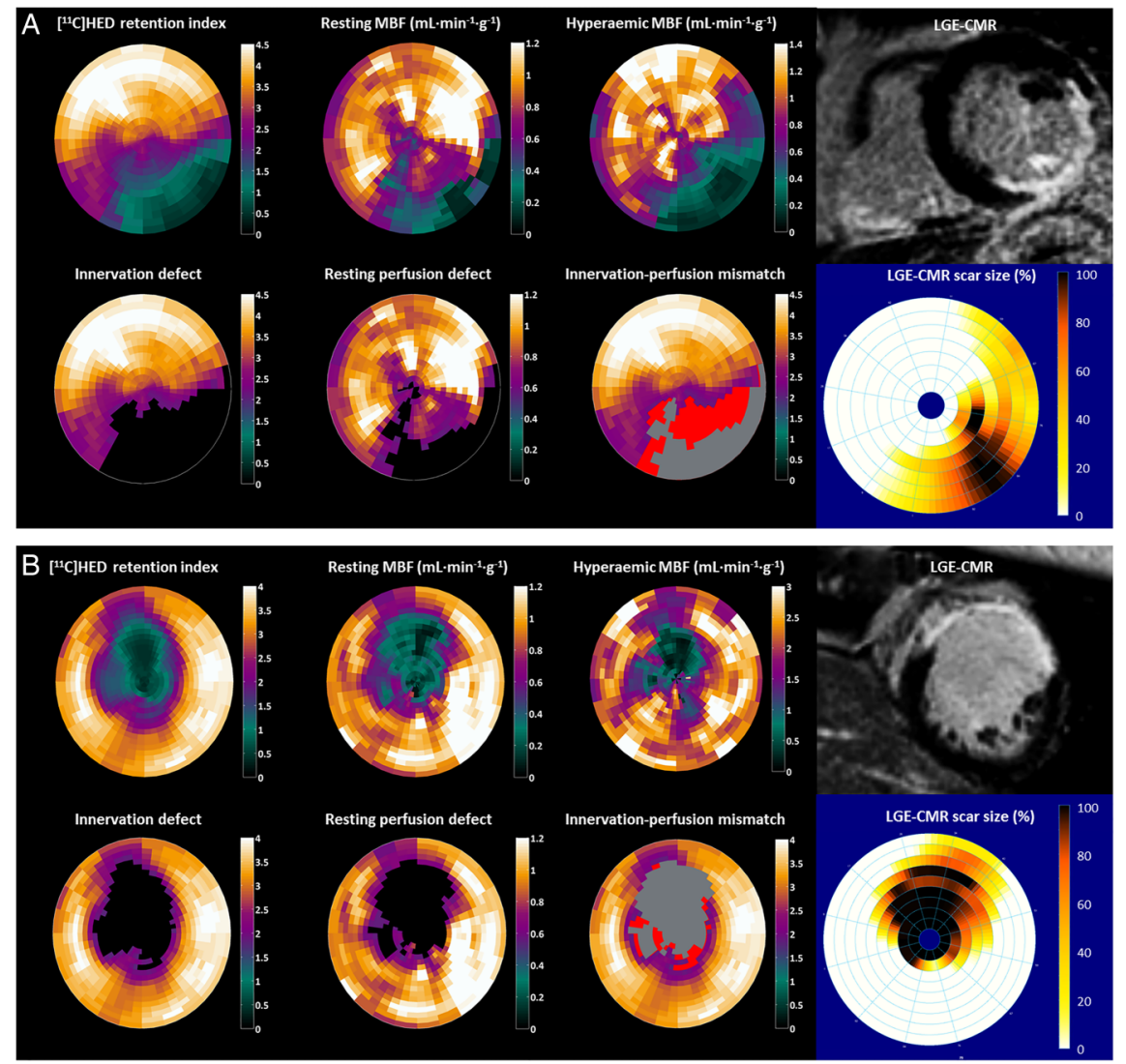

Figure 2 Examples of positron emission tomography (PET) and late gadolinium enhanced-cardiovascular MRI (LGE)-CMR results in two patients with electrophysiological study (EPS)-positive (A) and EPS-negative (B) result. (A) A patient with LGE scar in the inferolateral wall with corresponding PET perfusion and innervation defects, and significant innervation-perfusion mismatch as depicted in red. Quantitative PET revealed a severely impaired hyperaemic myocardial blood flow (MBF). (B) A patient with LGE scar in the anteroseptal wall with corresponding PET perfusion and innervation defects, and limited area of innervation-perfusion mismatch (red area). Quantitative PET revealed a relatively preserved hyperaemic MBF. 
Table 1 Multivariable predictors of coronary death ( $n=8762,233$ coronary deaths)

\begin{tabular}{|c|c|c|}
\hline Variable & HR $(95 \% \mathrm{CI})$ & p Value \\
\hline Age (per 10 years) & 2.33 (2.05 to 2.65$)$ & $<0.0001$ \\
\hline \multicolumn{3}{|l|}{ Sex } \\
\hline Female & 1 & $<0.0001$ \\
\hline Male & 1.91 (1.45 to 2.52$)$ & \\
\hline \multicolumn{3}{|l|}{ Character symptoms } \\
\hline Atypical & 1 & 0.0043 \\
\hline Typical & 1.59 (1.20 to 2.12$)$ & \\
\hline Non-cardiac & $1.05(0.67$ to 1.63$)$ & \\
\hline $\begin{array}{l}\text { Pulse rate (per } \\
10 \text { bpm) }\end{array}$ & 1.22 (1.13 to 1.33$)$ & $<0.0001$ \\
\hline \multicolumn{3}{|l|}{ Current smoker } \\
\hline No & 1 & 0.0016 \\
\hline Yes & 1.64 (1.21 to 2.23$)$ & \\
\hline \multicolumn{3}{|l|}{ Diabetes (y/n) } \\
\hline No & 1 & $<0.0001$ \\
\hline Yes & 1.99 (1.46 to 2.70$)$ & \\
\hline \multicolumn{3}{|l|}{ ECG normal } \\
\hline Normal & 1 & $<0.0001$ \\
\hline Abnormal & 1.96 (1.49 to 2.59$)$ & \\
\hline
\end{tabular}

clinical trial data to support that any of these tests alone or in combination should be used to efficiently guide ICD therapy and therefore none of the major societies have specifically endorsed any of these approaches".

The diagnosis of coronary disease at first presentation of suspected angina remains challenging, leading to costly diagnostic testing in many patients with a low likelihood of disease. Sekhri and colleagues (see page 869) assessed the value of simple clinical measures for prediction of coronary deaths over 10 years of follow-up in a multicenter cohort of 8762 patients with suspected angina (table 1). By including pulse rate, smoking status, diabetes and ECG findings, the proposed prognostic model was superior to the standard Diamond-Forrester model which includes only age, sex and symptoms (figure 3). Readers may want to try the online Prognosis In Suspected Angina (PISA) calculator provided by the authors at this website https://www. sealedenvelope.com/trials/pisa/

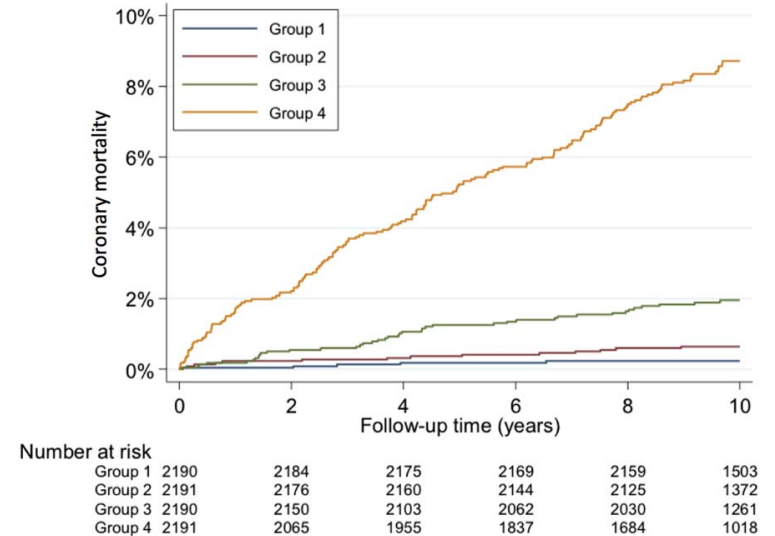

Figure 3 Kaplan-Meier cumulative coronary mortality by quarters of risk for the full prognostic model (based on table 1). There were 5, 14, 41, and 173 coronary deaths in risk groups 1 (lowest risk quarter) to 4 (highest risk quarter), respectively.

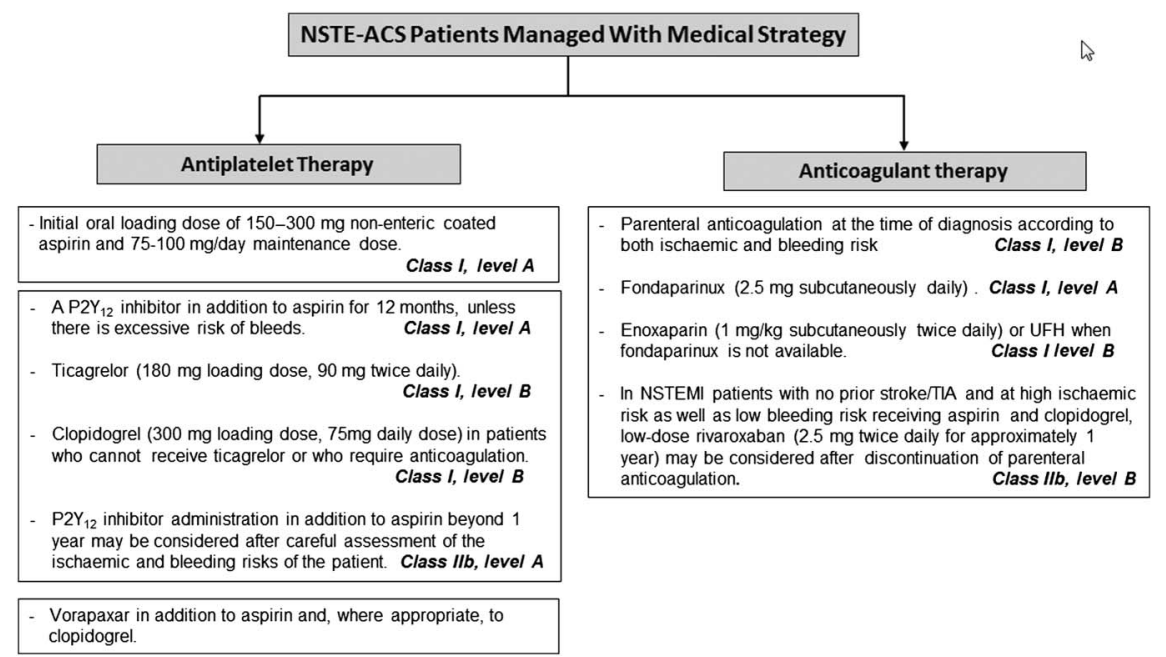

Figure 4 A decision algorithm of antithrombotic therapy in medically managed patients with NSTE-ACS. NSAID, non-steroidal anti-inflammatory drug; NSTE-ACS, non-ST-segment elevation acute coronary syndromes; NSTEMI, non-ST-segment elevation myocardial infarction; TIA, transient ischaemic attack; UFH, unfractionated heparin.

The Education in Heart article in this issue (see page 882) reviews antithrombotic therapy in medically managed patients with a non-ST elevation acute coronary syndromes. The primary learning objectives for this article include the ability to explain the mechanisms of action, potential side effects, and current guidelines relevant to the pharmacological agents used in treatment of this condition (figure 4).

This issue's Image Challenge (see page 881 ) shows an unusual echocardiographic finding that is important to know about be sure to look at the online videos, using the loop feature in your video viewer.

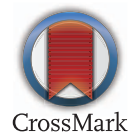

To cite Otto CM, Rahimi K. Heart 2016;102:811812.

Heart 2016;102:811-812.

doi:10.1136/heartjnl-2016-309848 